\title{
CONTRIBUTION OF BLOOD BIOMARKERS TO THE DIAGNOSIS OF CARDIOEMBOLIC STROKE
}

\author{
Markus Kneihsl (MD)a , Egbert Bisping (MD) ${ }^{b}$, Reinhard Raggam (MD)c, Christian Enzinger (MD)a,d, Simon \\ Fandler (MD)a , Sebastian Eppinger (MD)a , Kurt Niederkorn (MD)a , Harald Mangge (MD)e, Franz Fazekas \\ $(\mathrm{MD})^{a}$ and Thomas Gattringer (MD, PhD) ${ }^{a}$ \\ a Department of Neurology, Medical University of Graz, Graz, Austria \\ b Division of Cardiology, Department of Internal Medicine, Medical University of Graz, Graz, Austria \\ c Division of Angiology, Department of Internal Medicine, Medical University of Graz, Graz, Austria \\ d Division of Neuroradiology, Vascular and Interventional Radiology, Department of Radiology, Medical University of Graz, Graz, \\ Austria \\ e Clinical Institute of Medical and Chemical Laboratory Diagnostics, Medical University of Graz, Graz, Austria
}

\section{Background and Purpose}

Cardioembolic strokes can be effectively prevented by anticoagulation ${ }^{1}$. Blood biomarkers helping to suggest cardioembolic etiology and to steer the complex diagnostic workup would therefore be of great advantage ${ }^{2}$. This study addresses the possible contribution of biomarkers associated with heart failure (NT-proBNP) and thromboembolism (D-dimer) to the diagnosis of cardioembolic stroke.

\section{Methods}

Over a seven months period, we prospectively investigated all ischemic stroke patients admitted to our Stroke Unit for this study. All patients underwent a complete stroke work-up including cerebral imaging (CT and/or MRI), neurosonography, electrocardiography, cardiac rhythm monitoring (for at least 24 hours) and echocardiography. Blood to determine NT-proBNP and D-dimer levels was drawn within 24 hours after admission.

\section{Results}

Of 188 ischemic stroke patients (age: $69 \pm 14$ years, female: $38 \%$ ), 67 had cardioembolic (36\%), 73 non-cardioembolic (49\%) and 48 cryptogenic strokes (25\%), based on extensive work-up. NT-proBNP and D-dimer levels were significantly higher in cardioembolic vs. non-cardioembolic strokes (2543 vs. 707 $\mathrm{pg} / \mathrm{ml}, \mathrm{p}<0.001 ; 2.4$ vs. $1.4 \mu \mathrm{g} / \mathrm{ml}, \mathrm{p}<0.001)$. The area under the curve (AUC) of NT-proBNP obtained for the diagnosis of cardioembolic stroke was 0.85 (95\% Cl: 0.78-0.92). The cut-off point with the highest sensitivity and specificity was identified at $525 \mathrm{pg} / \mathrm{ml}$ (sensitivity: 82\%, specificity: 77\%; Figure 1). The AUC of D-dimer in cardioembolic stroke was $0.69(95 \% \mathrm{Cl}: 0.60-$ 0,79 ) with a cut-off of $0.75 \mu \mathrm{g} / \mathrm{ml}$ (sensitivity: $67 \%$, specificity: $69 \%$; Figure 2).

${ }^{1}$ Freedman B, Potpara TS, Lip GY. Stroke prevention in atrial fibrillation. Lancet. 2016 388: 806-817.

${ }^{2}$ De Marchis GM, Schneider J, Weck A, et al. Midregional proatrial natriuretic peptide improves risk stratification after ischemic stroke. Neurology. 2018; 90: e455e465.

Abbrevations

CE: Cardioembolic stroke; LVD: Large vessel disease; SVD: Small vessel disease CS: Cryptogenic stroke

\section{Conclusions}

In concordance with previous studies, NT-proBNP has reasonable diagnostic accuracy for stroke related to cardiac embolism. Nevertheless, sensitivity and specificity are too low to essentially improve the diagnostic work-up. The contribution of D-dimer levels is even more limited.

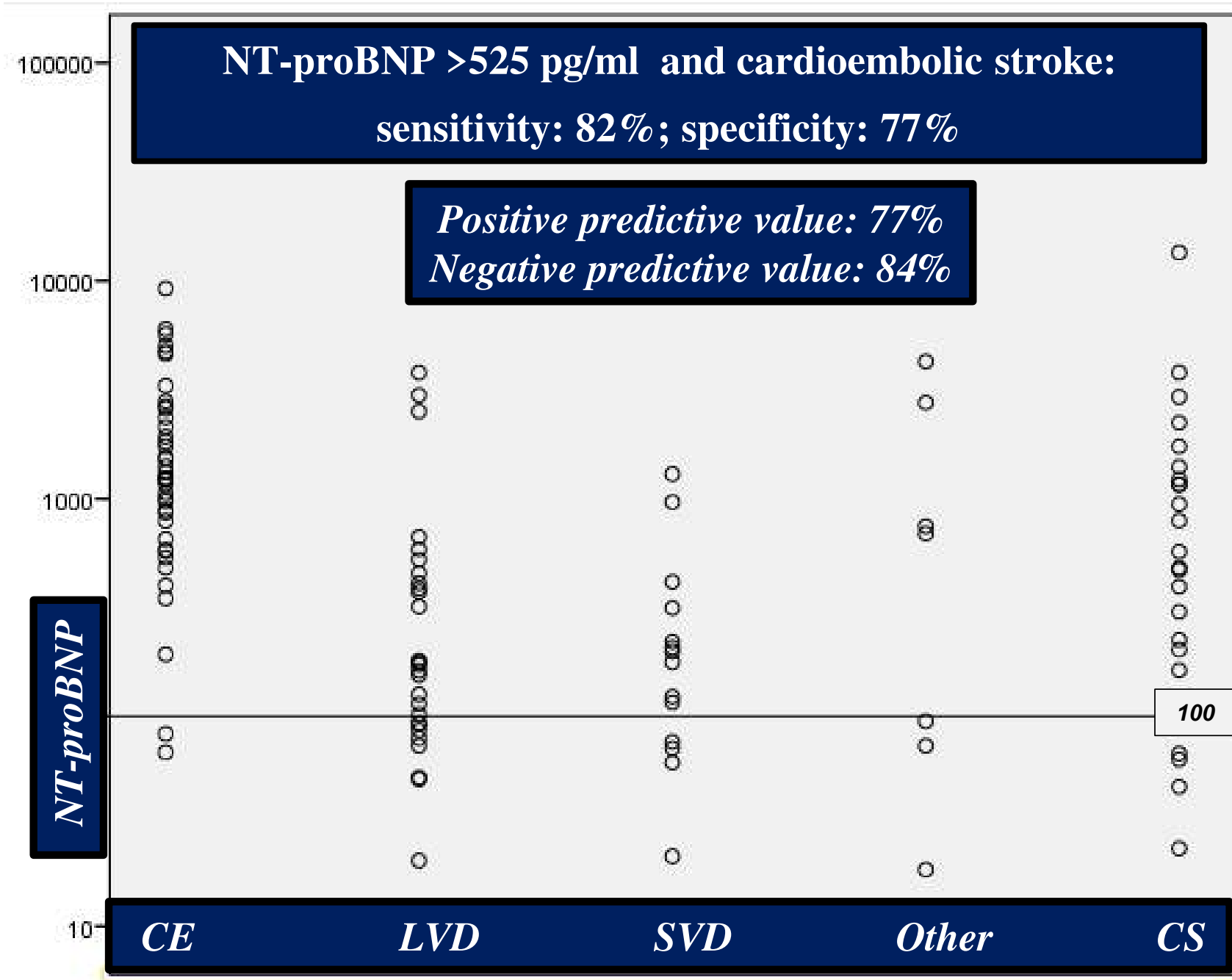

Figure 1. Cloud chart of NT-proBNP and stroke subtypes

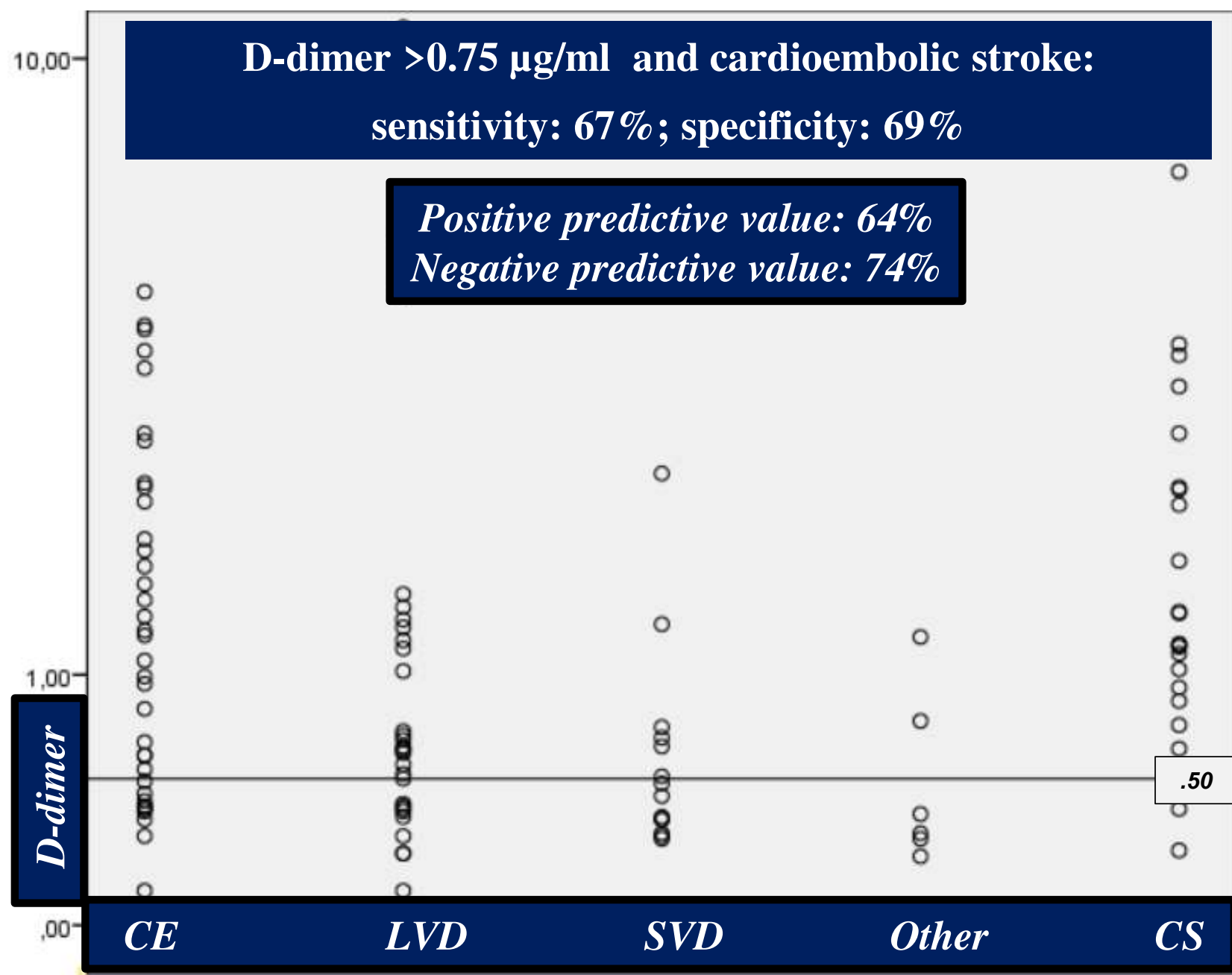

Figure 2. Cloud chart of D-dimer and stroke subtypes 